\title{
Hyperdense object in mandibular fracture line
}

\author{
Nils H Rohleder \\ Department of Oral and Maxillofacial Surgery, Technical University of Munich, School of Medicine, Ismaninger Str. 22, Munich, Germany
}

A healthy 35-year-old woman fell off her mountain bike during a tour through the Bavarian forest when the floor became slippery because of an upcoming storm. She fell on her chin and presented in the maxillofacial unit of the Technical University of Munich, Germany, with pain in the anterior mandible and pre-auricular region on both sides. A small laceration of 0.2 inches without bleeding was visible between the first and the second incisor in the left mandible. Dental panoramic X-ray and a CT scan revealed fractures of both condyles and the anterior mandibular body, with a hyperdense object located in the anterior fracture line (Figure 1, panels A and B, arrows), which could not be identified by the radiologists. The object was recovered during the operative treatment of the patient (open reduction, internal fixation of the anterior fracture). It was identified as an amalgam dental filling dislocated from the first molar tooth in the left maxilla, which was broken during the accident. This is quite notable because the filling took a very unlikely way from the distal aspect of the maxilla into the

Copyright: (2019 Rohleder NH. This is an open-access article distributed under the terms of the Creative Commons Attribution License, which permits unrestricted use, distribution, and reproduction in any medium, provided the original author and source are credited. small gingival laceration and down into the fractur line of the anterior mandible. The patient recovered completely. The tooth which lost the amalgam filling was treated conservatively with a composite filling.

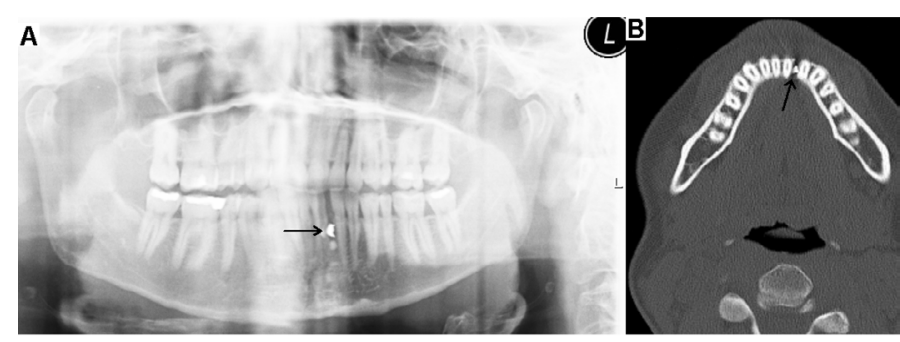

Figure 1. Dental panoramic radiography shows an unidentified hyperdense object in projection of the mandibular fracture line (A, indicated by arrow). Axial CT scan confirms the object to be located directly in the fracture line (B, indicated by arrow). 